\title{
TPF-I Planet Detection Testbed: Progress in Testing Exo-planet Signal Detection
}

\author{
S. R. Martin, P. Szwaykowski and F. M. Loya \\ Jet Propulsion Laboratory, 4800 Oak Grove Dr, Pasadena, CA, USA \\ email: stefan.r.martin@jpl.nasa.gov
}

\begin{abstract}
The Planet Detection Testbed is designed to simulate the architecture and operation of a space-borne four-telescope nulling interferometer. Constructed in the form of a dual chopped Bracewell interferometer together with star and planet sources, it reproduces the principal features of the flight beam combiner designed at JPL for the proposed TPF-I Formation Flying Interferometer. The aims of the testbed are to demonstrate stable four-beam nulling and planet detection at representative star-planet contrast ratios. In the flight design, starlight between 7 and 17 micron wavelength is to be nulled, and 2 to 3 micron starlight is used for fringe tracking and phasing the interferometer. There are also metrology systems and alignment systems which are required for deep and stable nulling. The testbed reproduces these features; 2 to 3 micron light from a thermal source is used for fringe tracking, and nulling and planet detection is performed at 10 microns. The testbed also incorporates laser metrology and other systems enabling continuous control of beam alignment. The ultimate goal is to simulate planet detection at star-planet contrast ratios of order $10^{-7}$ during full rotations of the telescope array using the phase chopping method. The latest results from the testbed are presented including four-beam nulling experiments at null depths of $10^{-5}$ and planet signal detections at similar contrast ratios.
\end{abstract}

Keywords. Nulling, Stellar Interferometry, Exoplanet.

\section{Introduction}

The detection of extra-solar planets using the radial velocity technique, more than one hundred so far, for example Marcy et al. (2005), and more recently the direct optical detection using the NACO instrument (Chauvin et al. (2004)) of a giant planet orbiting some distance from its parent star is spurring efforts to detect earth-like planets nearer their stars, within the habitable zone, where temperatures would be conducive to the existence of life. After the challenge of detection amidst the glare from the bright parent star, the characterization of the planet using spectroscopy will require long integrations and stable operation of the telescope. In the thermal infrared between 7 and $20 \mu \mathrm{m}$, absorption bands for gases considered to be markers for life processes occur; the thermal spectrum of the Earth viewed from space for example, is modified by the presence of water, methane, ozone and carbon dioxide absorption features in the atmosphere. In the case of the thermal infrared, an earth-like planet orbiting within the habitable zone of a G0 star at a distance of $10 \mathrm{pc}$ has an intensity contrast with the star of a few $10^{-7}$ and a very small angular separation. Some means of suppressing the bright starlight together with a wide telescope collecting aperture would be helpful in separating the planet light from the star light. To achieve a detection, the concept of a 4-aperture telescope employing $4 \mathrm{~m}$ diameter collecting apertures with a baseline length between 60 and almost $500 \mathrm{~m}$, floating in an earth-trailing orbit is being studied. Such a device, the Terrestrial Planet Finder Interferometer (TPF-I), will employ the method of nulling interferometry (Wallace et al. (2000)) to suppress the bright starlight and enable the planet photons to 
be detected and then characterized. To detect the planet will require observation times of 10 hours or more, with greater times needed for spectroscopic measurements. The Planet Detection Testbed is being used to study the combination of beams of infrared light from four telescopes, with an emphasis on achieving deep, stable nulls and performance near to that which will be required for successful operation in space.

\section{The Planet Detection Testbed}

The Planet Detection Testbed has been described elsewhere (Martin, S.) and consists of a pair of nulling beamcombiners and a cross-combiner configured for nulling and phasechopping four input beams. The testbed sources simulate a star and a faint planet. The starlight which is to be nulled is produced by a $\mathrm{CO}_{2}$ laser operating near $10.6 \mu \mathrm{m}$. A ceramic filament also provides broadband radiation which is used for fringe tracking, so that the interferometer can be accurately phased on the star. The fringe trackers operate at various wavelengths between about 2.3 and $3.5 \mu \mathrm{m}$. The planet source is also a ceramic filament with a dichroic filter to narrow the output to the waveband between 10 and $11 \mu \mathrm{m}$. The star and the planet sources are passed through separate choppers so that they can be more readily detected using lock-in amplifiers connected to the output of $\mathrm{HgCdTe}$ and InSb infrared detectors. To form the four input beams, the starlight is split once using a broadband 50/50 beamsplitter, and then each beam is split again. The planet light is similarly split once, and then combined with the starlight at the second splitter. This combination method ensures that the planet light has a certain phase relative to the star causing it to be constructively interfered at the nulling beamsplitter when the star is nulled. The recombination of the beams follows the splitting process in reverse; first, pairs of beams are nulled, and then they are combined on the cross-combiner beamsplitter.

\section{Null Stabilization}

It can be shown that to achieve deep nulls the pairs of input beams must be very similar in terms of intensity and phase. In essence, the aim is to achieve equal electric field amplitudes from each beam at the nulling detector, and carefully control the phase or optical path for each beam. For small deviations from perfect alignment, for example tilt or shear of the beams, the effect is to reduce the coupling to the nulling detector and therefore degrade the null. So, if beam tilt and shear are well controlled, barring other aberrations, only beam optical path remains to be stabilized. A control loop maintains the ideal optical path by means of a lock-in amplifier loop which monitors the nulling signal for an imposed dither signal. The sinusoidal dither signal is placed on the optical path of one of the incoming beams by modulating a piezoelectric (PZT)-driven stage so that the optical path change is approximately one nanometer. When the interference fringe is a small distance away from null the dither frequency appears on the nulling detector output with a phase dependent on whether the optical path difference of the interfering beams is positive or negative. The lock-in control loop software mixes the incoming detector output with the drive signal to the PZT and integrates the result to form an integral error signal. If the result is positive the loop drives the PZT in one direction and if negative it drives the PZT in the opposite direction. With an appropriate choice of loop coefficients the result is a fringe that is stabilized at null or alternatively at the peak. This control technique has been successfully used in previous experiments (Martin et al. (2003)) to achieve null depths of one million to one in a smaller infra-red nulling testbed. In the Planet Detection Testbed the optical path is comparatively long, 10 meters, and the beams strike a number of mirrors as they pass from the input to the detector. The optical 


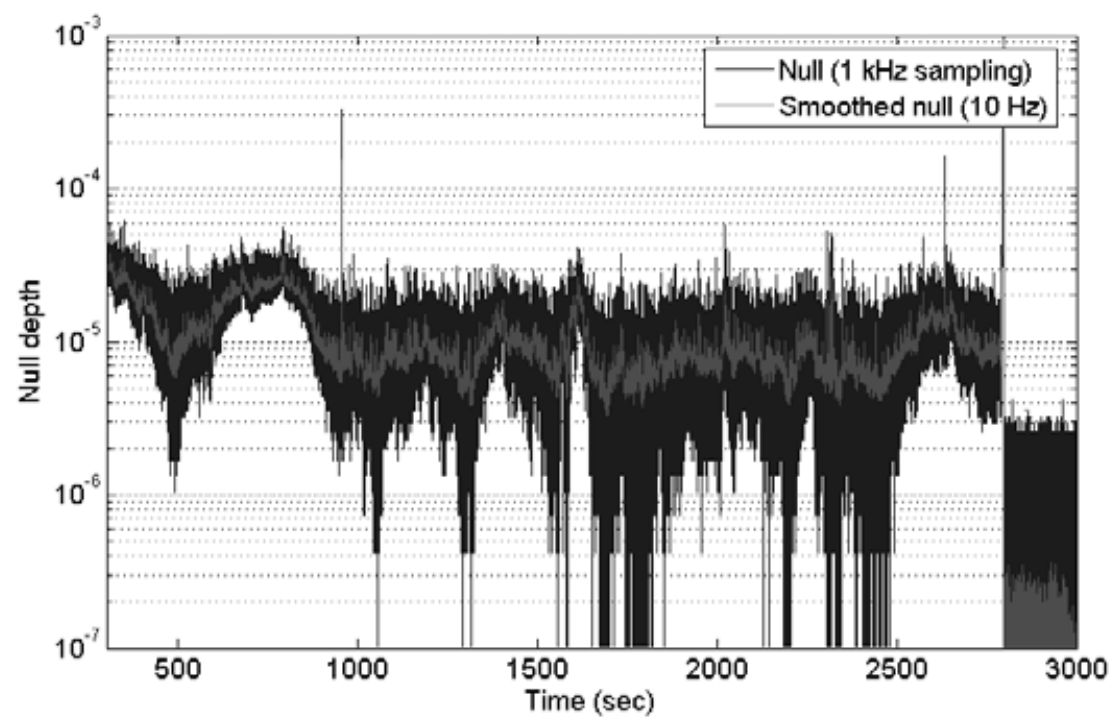

Figure 1. Nulling data.

alignment is subject to drifting because of small mechanical and thermal changes and it was found experimentally that without control of alignment, null depths greater than 10,000 are unlikely to be maintained for more than a few minutes. The drifting of optical alignment also produced a second problem for testbed operations: to emulate TPF-I operation the testbed phase must be maintained by a fringe tracker, operating on two to three micron radiation. This radiation is picked up by detectors positioned on either side of each nulling beamsplitter and on either side of the cross-combiner beamsplitter and the relative intensity of the light on each side of the beamsplitters is used as a measure of the phase. When the beams tilt, the intensity at the detector is affected and an error is introduced into the phase measurement. Because this effect was present in the unstabilized testbed, the fringe tracker could not hold the null fringe precisely although it would remain somewhere near the fringe.

To overcome this problem and to enable deep nulls to be maintained, a two-component dither loop was employed to control the tilt and tip of one of each pair of beams. In the experiments detailed below, two of the incoming beams (beams 1 and 3) were directed at the nulling detector and their directions and phases were not closely controlled. A second beam (beams 2 and 4 respectively) is paired with each of these incoming beams, and dither signals are applied to the second beam to enable control of its phase and angle relative to its partner. To control the beam angle the lock-in method was again used. A circular dither was applied to a tip/tilt stage in beam 2 or 4 so that the angular dither in the horizontal direction would be sinusoidal (for example) and the angular dither in the vertical direction would be cosinusoidal. The effect of the circular dither is to modulate the nulling output, and when the two beams are coaligned the null fringe is at a minimum. Again, the phase of the dither signal can be used to determine the adjustment direction and the tip/tilt control loop can drive the alignment of the pair of beams to an optimum via a simple integral loop.

The next stage in the nulling process is to locate the correct fringe. Since a broadband source is needed for this operation, the fringe tracking light is used to locate the correct 
optical path setting. The PZT stage is driven over the full extent of its range and the fringe tracker signal analyzed to locate the center of the fringe. Two methods by which this might be located (Pedretti et al. (2004)) were tested but it was found that they did not very reproducibly find the desired location on the fringe, possibly because of small alignment changes. Therefore a simple peak detection routine was used instead and this reliably selected a position near the center of the fringe.

Once this location is found the stage is driven to the fringe center and then a dither loop is engaged to drive the interferometer down to the bottom of the null. Once there the fringe tracker loop is engaged to hold the null approximately by maintaining set amplitudes on the pair of fringe tracking detectors placed either side of the nulling beamsplitter. At this stage a shutter can be closed to exclude the residual light from this nulling beamsplitter from the nulling detector and the second nuller can be phased. Once the second nuller is nulling, the shutter can be reopened, the fringe tracking loop discontinued, the dither loop restarted, and the system is now nulling four beams simultaneously. To reach deep nulls the beam alignment must be corrected for any drift and fine-tuned, so the circular dither loop is engaged for beams 2 and 4 . When all the loop coefficients have been correctly chosen the nuller is driven below a 100,000 null depth. At the null, four loops are running, $8 \mathrm{~Hz}$ and $12 \mathrm{~Hz}$ circular dithers to maintain the angular alignments, and $20 \mathrm{~Hz}$ and $30 \mathrm{~Hz}$ linear piston dithers to maintain the optical paths. These signals all impose small modulations on the nulling detector output.

\section{Results}

To reach one of our development milestones, a faint planet source at 100000:1 contrast ratio with the star was to be detected while nulling at 100000:1 null depths. The planet could be detected by any reasonable means in the presence of the bright starlight; amplitude chopping of the planet at its source was employed as the detection mode. At the cross-combiner the planet light is incoherently summed; the nullers are not cophased and so the measured input planet light may be easily compared with the output data.

Once nulling deeply on all four beams the trace shown in Figure 1 was obtained. The blue trace shows the raw data (sampling rate $1 \mathrm{kHz}$ ) and the red trace the smoothed data (time constant $10 \mathrm{~Hz}$ ). At about $970 \mathrm{~s}$, the planet signal was admitted into the system; between about $900 \mathrm{~s}$ and $2500 \mathrm{~s}$ the mean null depth is below $10^{-5}$. The apparent noisiness of the trace is mainly due to the control loops which modulate the null at various frequencies. There was also a significant amount of disturbance caused by vehicular traffic outside the building.

The various loops run at these frequencies; 20 and $30 \mathrm{~Hz}$ for OPD, and 8 and $12 \mathrm{~Hz}$ for tip/tilt. Prominent sum and difference signals are expected at $10 \mathrm{~Hz}$ and $50 \mathrm{~Hz}$, and weaker ones at 40 and $60 \mathrm{~Hz}$, with other weaker signals. The planet signal modulation was applied by running the planet chopper at a frequency some $70 \mathrm{~Hz}$ below the star chopper. In practice, the frequency difference drifted continuously over several $\mathrm{Hz}$ owing to the poor stability of the chopper drivers.

Samples were taken from the trace at 860 to $960 \mathrm{~s}$ (a section with no planet), 1100 to $1400 \mathrm{~s}$ (a section with a weak planet signal), and 1700 to $2400 \mathrm{~s}$ (a section with a stronger planet). These samples were analyzed by taking 1 second time intervals and calculating the magnitude of the Fourier transform for each interval, then summing and obtaining the mean spectrum in the form of a spectral estimate.

Figure 2 shows the results; the red line shows the signal without the planet, the blue line shows the stronger planet and the green the weaker planet signals. The units of the $y$ axis are contrast ratio with the star. The broadening of the planet signal by the 


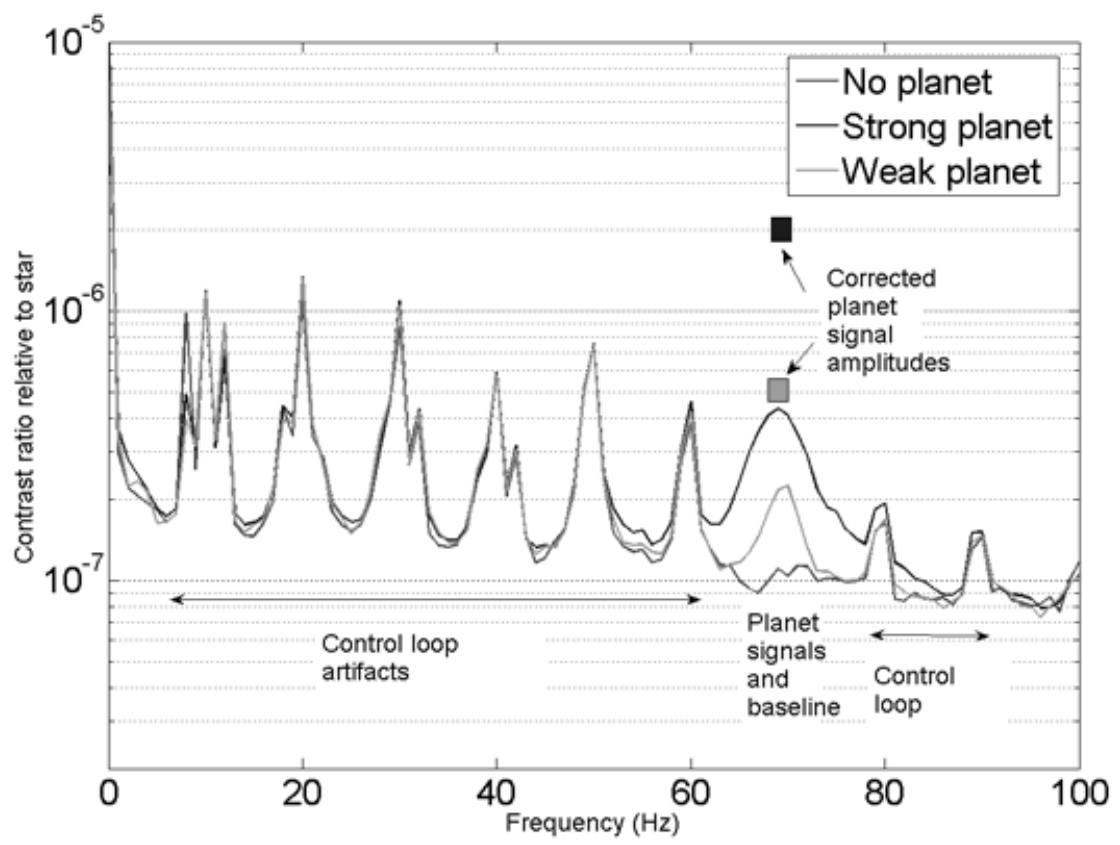

Figure 2. Spectrum of signal from nuller showing the control loop artifacts and the planet signal at $70 \mathrm{~Hz}$.

frequency instability reduces the apparent planet contrast below its true value. To get a better estimate of the signal amplitude the planet signal was integrated over a $7 \mathrm{~Hz}$ bandwidth. Then, for the stronger signal, the amplitude is $2.7810^{-6}$, and for the weaker signal it is $1.3210^{-6}$. The noise amplitude in the same passband is $8.210^{-7}$.

Subtracting the background noise, the ratio of the strong to the weak planet signal is 3.9 which agrees well with direct measurements of the planet signals in the absence of the star source. These measurements showed that the strong planet signal intensity would produce $1.2310^{-5} \mathrm{~V}$ at the detector, and the weak signal $2.7610^{-6} \mathrm{~V}$, a ratio of 4.5. The planet to star contrast ratio from the direct measurements (strong signal) would be expected to be a little less than $1.9710^{-6}$ which agrees well with the spectrally estimated value of $1.9610^{-6}$.

The mean null depth over the period 1700 to $2460 \mathrm{~s}$ was $1.3710^{5}: 1$. The star:planet contrast at the strong setting was $5.110^{5}: 1$, comfortably exceeding the $10^{5}: 1$ goal, and at the weak setting it reaches $2.010^{6}: 1$.

\section{Future plans}

The next steps in testbed development require deeper nulls of order $10^{-6}$, cophasing of the cross combiner, and planet detection at better than $10^{-6}$ contrast ratio with the star. Phase chopping will be employed at the crosscombiner. To achieve the deeper nulls additional stabilization equipment will be added to the testbed in the form of a laser beam coaligned with the starlight which will be detected by shear and pointing detectors located immediately prior to the nulling beamsplitter. Pointing control to better than 0.01 arc sec and shear control to better than 1 micron across a $25 \mathrm{~mm}$ diameter beam have 
been achieved in testing, which will be adequate for our needs. The second modification for deeper nulling will be the use of a laser OPD metrology system to operate in concert with the fringe tracker, replacing the dither loop now controlling the optical path. The metrology system is needed to compensate for the high-speed vibrations arising from the environment and from the mechanical choppers on the testbed that cannot be followed by the fringe tracker.

\section{Acknowledgements}

The authors would like to acknowledge the contributions of the other members of the testbed team: Kurt Liewer, Hong Tang, Randall Bartos, Steve Monacos, Raymond Lam, Boris Lurie and Nasrat Raouf. The work reported here was conducted at Jet Propulsion Laboratory, California Institute of Technology, under contract with NASA. Reference herein to any specific commercial product, process, or service by trade name, trademark, manufacturer, or otherwise, does not constitute or imply its endorsement by the United States Government or the Jet Propulsion Laboratory, California Institute of Technology.

\section{References}

Chauvin, G., Lagrange, A.-M., Dumas, C., Zuckerman, B., Mouillet, D., Song, I., Beuzit, J.-L., \& Lowrance, P. 2004, Astronomy and Astrophysics 425, L29-L32

Marcy, G. W., Butler, R. P., Vogt, S. S., Fischer, D. A., Henry, G. W., Laughlin, G., Wright, J. T., \& Johnson, J. A., 2005, Ap.J, 619, 570

Martin, S., 2005, 2005 IEEE Aeospace Conf "TPF Planet Detection Testbed"

Martin, S. R., Gappinger, R. O., Loya, F. M., Mennesson, B. P., Crawford, S. L., \& Serabyn, E., 2003, Proc. SPIE 5170, 144-154

Pedretti, E., Thureau, N. D., Wilson, E., Traub, W. A., Monnier, J. D., Ragland, S., Carleton, N. P., Millan-Gabet, R., Schloerb, F. P., Brewer, M. K., Berger, J.-P., \& Lacasse, M. G., 2004, Proc. SPIE 5491, 540-550

Wallace, K., Hardy, G., \& Serabyn, E., 2000, Nature 406, 700 